\title{
Electronic structure of charge and spin stripe order in $\mathrm{La}_{2-x} \mathrm{Sr}_{x} \mathrm{NiO}_{4}\left(x=\frac{1}{3}, \frac{1}{2}\right)$
}

\author{
Susumu Yamamoto, ${ }^{1}$ Takeo Fujiwara, ${ }^{2,1}$ and Yasuhiro Hatsugai ${ }^{3,4}$ \\ ${ }^{1}$ Core Research for Evolutional Science and Technology, Japan Science and Technology Corporation (CREST-JST), Japan \\ ${ }^{2}$ Center for Research and Development of Higher Education, University of Tokyo, Tokyo 113-8656, Japan \\ ${ }^{3}$ Institute of Physics, University of Tsukuba, 1-1-1 Tennodai, Tsukuba, Ibaraki 305-8571, Japan \\ ${ }^{4}$ Department of Applied Physics, University of Tokyo, 7-3-1 Hongo, Bunkyo-ku, Tokyo 113-8656, Japan
}

(Received 15 August 2007; published 11 October 2007)

\begin{abstract}
Electronic structure of stripe ordered $\mathrm{La}_{2-x} \mathrm{Sr}_{x} \mathrm{NiO}_{4}$ is investigated. The system with $x=\frac{1}{3}$ is insulator, in LSDA $+U$ calculations, and shows charge and spin stripe, consistent with the experimental results. A highly correlated system of $x=\frac{1}{2}$ is studied by using exact diagonalization of the multiorbital many-body Hamiltonian derived from LDA calculations and including on-site and intersite Coulomb interactions. The fluctuation of the residual spin on $\mathrm{Ni}^{3+}$ (hole) site couples with the charge fluctuation between $\mathrm{Ni}^{3+}$ and $\mathrm{Ni}^{2+}$ states and this correlation lowers the total energy. The resultant ground state is an insulator with charge and spin stripe of the energy gap $0.9 \mathrm{eV}$, consistent with the observed one. The on-site Coulomb interaction stabilizes integral valency of each $\mathrm{Ni}$ ion $\left(\mathrm{Ni}^{3+}\right.$ and $\left.\mathrm{Ni}^{2+}\right)$, but does not induce the charge order. Two quantities, intersite Coulomb interaction and anisotropy of hopping integrals, play an important role to form the charge and spin stripe order in a system of $x=\frac{1}{2}$.
\end{abstract}

DOI: 10.1103/PhysRevB.76.165114

PACS number(s): 72.80.Ga, 71.27.+a, 71.45.Lr, 75.30.Fv

\section{INTRODUCTION}

The stripe order of charge and spin has been found in several layered perovskites ${ }^{1-3}$ and organic conductors. ${ }^{4,5}$ Both of them are of the pseudo-two-dimensional (2D) electron system and have strong Coulomb interactions compared to hopping integrals. A single band system can be a model of organic conductors, while, in layered perovskites, a number of relevant orbitals depends on filling, crystal field, exchange splitting, etc. The intersite Coulomb interaction is essential to the charge stripe in organic conductors, and then the extended Hubbard model is adopted to explain the mechanism of stripe order there. ${ }^{6}$ In layered perovskites, however, an origin of the stripe order is still controversial particularly in the perovskites other than cuprate, while the order in cuprate is attracting much attention in conjunction with marked suppression of $T_{c}$ with hole doping $\frac{1}{8}$ and rich physics.

Nickel compound $\mathrm{La}_{2-x} \mathrm{Sr}_{x} \mathrm{NiO}_{4}$ (LSNO) is a typical system of static stripe order of charge and spin. ${ }^{7-10}$ It is an insulator with the total spin $S=0$ in a wide range of $\mathrm{Sr}$ doping $x(0 \leqslant x \leqslant 0.9) .{ }^{11}$ This stability of insulating phase is quite different from the variety of the phases in the cuprate case; cuprate changes to metal, insulator, and superconductor depending on the hole concentration. LSNO at $x=\frac{1}{3}$ shows the highest spin-order temperature $T_{\mathrm{SO}} \sim 200 \mathrm{~K}$, because stripe order of charge and spin is commensurate with lattice periodicity. The periods of charge and spin stripe are not generally commensurate to the lattice. Incommensurability $\epsilon$ increases with increasing $x$ and satisfies $\epsilon \gtrsim x$ in the region $\frac{1}{3}>x>0, \epsilon \lesssim x$ in the region $x>\frac{1}{3}$. Here, incommensurability $\epsilon$ is defined as displacement of peak positions of super structure from reciprocal lattice points. Increase of $\epsilon$ saturates in the region of $\frac{1}{2}>x>\frac{1}{3}$ with the value $\epsilon \sim 0.44 .{ }^{9}$ In the region $x \geq \frac{1}{2}$, there exists a commensurate charge ordered phase without magnetic order, called a checkerboard-type charge order, between $T_{\mathrm{SO}}=80 \mathrm{~K}$ and $T_{\mathrm{CO}}=480 \mathrm{~K} \cdot{ }^{10}$ Another experimental fact in LSNO is the dependence of the ratio be- tween two lattice constants along with $c$ and $a$ axis $(c / a$ ratio) on $x$. The observed $c / a$ ratio has a maximum value at $x=\frac{1}{2}$, because holes are first doped into the $x^{2}-y^{2}$ orbital in a region $x<\frac{1}{2}$ and, then, additional holes are doped into the $3 z^{2}-1$ orbital when $x>\frac{1}{2} .11$

We present two issues on LSNO in this paper by using both LSDA $+U$ method and the exact diagonalization of a many-body Hamiltonian. The first issue is that the intersite Coulomb interaction is essential to static charge order in doped layered perovskites. Not only Hartree energy but also correlation energy due to the intersite Coulomb interaction (beyond Hartree-Fock approximation) is important. Because the electron configurations fluctuate between hole $\left(\mathrm{Ni}^{3+}\right)$ and nonhole $\left(\mathrm{Ni}^{2+}\right)$ states by hopping, the correlation energy is maximized at $x=\frac{1}{2}$. In this situation, we need to diagonalize the many-body Hamiltonian to know the true ground state at $x=\frac{1}{2}$. The second issue is that the spin stripe order in LSNO is attributed to the structure of multiorbitals. A spin moment on a hole site is strongly correlated with surrounding spin moments on nonhole sites in the multiorbital system with fractional occupation, which is a essential difference from the case of single-orbital systems.

The stripe order in layered perovskites is, in some cases, attributed to the Jahn-Teller (JT) distortion. ${ }^{12,13}$ In the present system, however, this is not the case, because the JT distortion is not consistent with the local symmetry of the observed stripe order of charge and spin in $x=\frac{1}{3}$ LSNO. In the $E_{g}$-type JT distortion coupling with the $e_{g}$ orbitals, two oxygen atoms at opposite positions, centering the $\mathrm{Ni}$ site, displace in opposite directions with each other. Consequently, the periodicity should be doubled along with the Ni-O-...$\mathrm{O}-\mathrm{Ni}$ line. It contradicts the observed tripled structure. Distortion with ungerade mode can be consistent with the observed order but increases the total energy. The spin structure at $x=\frac{1}{2}$ is inconsistent with the JT distortion, too.

There is another candidate for the origin of stripe order in layered perovskites. That is long-ranged Coulomb 
interaction. ${ }^{14}$ Also it is well known that the intersite Coulomb interaction stabilizes the charge order in the quarterly filled (single-orbital) extended Hubbard model. ${ }^{6,15}$ Thus, the intersite Coulomb interaction can stabilize the electronic structure of insulator with static stripe order. In the electronic structure of LSNO, the degeneracy of $3 d e_{g}$ orbitals causes two important parameters: splitting between $3 z^{2}-1$ orbital and $x^{2}-y^{2}$ orbital and the hopping integrals. It is reported that different values of these variables bring the system different order. ${ }^{13,16}$ We should get reliable values of these parameters from the first-principles electronic structure calculations.

The present paper is organized as follows. The charge and spin stripe of LSNO $\left(x=\frac{1}{3}\right)$ is discussed in Sec. II, based on LSDA $+U$ calculation. We show in Sec. III the problem that charge ordered solution is not found in LSDA $+U$ calculations. Then, we explain that the LSNO of $x=\frac{1}{2}$ is a highly correlated system and the correlation energy in LSDA $+U$ method is not enough to stabilize the charge ordered solution as a ground state. In later sections, exact diagonalization of the many-body Hamiltonian is employed to investigate the electronic structure of LSNO $\left(x=\frac{1}{2}\right)$. The Hamiltonian derived from LDA calculation is explained in Sec. IV. Section $\mathrm{V}$ is devoted to discussion of the charge stripe order and charge correlation functions in the system of $x=\frac{1}{2}$. The excitation spectra and the energy gap of the system with $x=\frac{1}{2}$ is discussed in Sec. VI. The spin stripe order of LSNO $\left(x=\frac{1}{2}\right)$ is discussed in Sec. VII. Finally Sec. VIII is the conclusion.

\section{CHARGE AND SPIN STRIPE OF LSNO $\left(x=\frac{1}{3}\right)$ BY USING LSDA $+U$ METHOD}

$\mathrm{LSDA}+U$ method $^{17,18}$ in conjunction with the linear muffin-tin orbital method with the atomic sphere approximation ${ }^{19,20}$ includes on-site Coulomb and exchange interaction $U, J$ with rotational invariant form. ${ }^{18,21}$ This onsite Coulomb term in LSDA $+U$ Hamiltonian is called a "Hubbard correction term." LSDA $+U$ also includes the intersite Coulomb interaction by means of Hartree energy. Therefore, LSDA $+U$ method can explain the physics of any charge ordered system, if Hartree energy is enough to describe them.

For low doping systems $\left(x=0, \frac{1}{3}\right)$ where the correlation energy induced by the inter-site Coulomb interaction is small, the results of LSDA $+U$ calculations can explain well experimental results. Details of the calculations are as follows. The values of $U$ are chosen to be $7.5 \mathrm{eV}$ for $\mathrm{Ni}^{2+}$ ions ${ }^{22}$ and to be $7.0 \mathrm{eV}$ for $\mathrm{Ni}^{3+}$ ions. ${ }^{23}$ The values of $J$ are chosen to be $0.88 \mathrm{eV}$. These values are consistent with those of constrained LDA. ${ }^{17}$ In the present system, $\mathrm{NiO}_{6}$ octahedra are elongated ${ }^{11}$ with the direction of the $c$ axis and tilted. ${ }^{24}$ The elongation splits the two $e_{g}$ orbitals by $\Delta=0.97 \mathrm{eV}$ in the LDA calculation, where we denote the energy splitting between $3 z^{2}-1$ orbital and $x^{2}-y^{2}$ orbital as $\Delta$. On the other hand, the tilt of $\mathrm{NiO}_{6}$ octahedra does not change the electronic structure in both LDA and LSDA $+U$ calculations; the localized magnetic moments on the $\mathrm{Ni}$ ion are $1.56 \mu_{B}$ (with tilt) and $1.54 \mu_{B}$ (without tilt), and the energy gap $3.66 \mathrm{eV}$ (with tilt) and $3.73 \mathrm{eV}$ (without tilt). Therefore, the tilt of

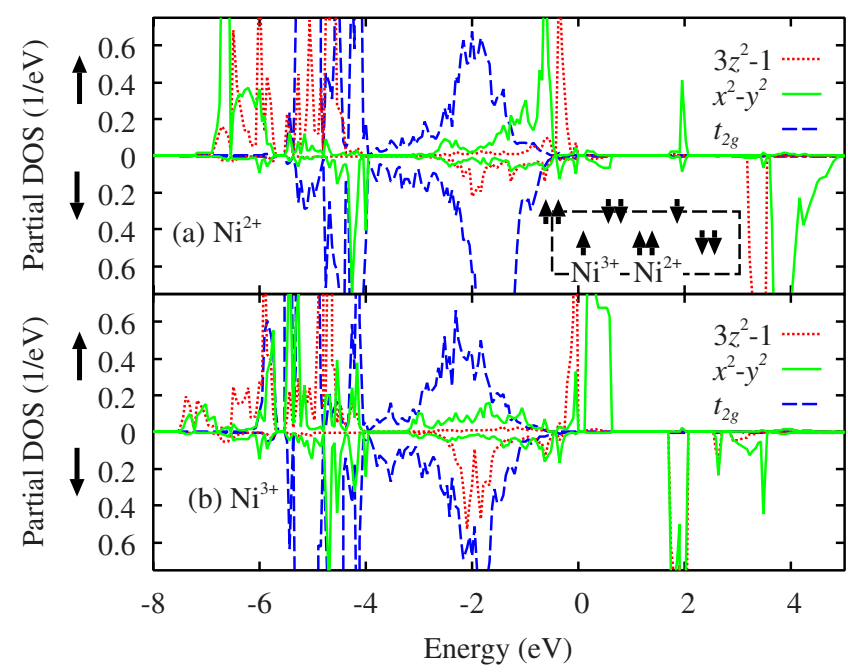

FIG. 1. (Color online) Projected density of states for each spin (up and down indicated by arrows) of electron in $\mathrm{La}_{5 / 3} \mathrm{Sr}_{1 / 3} \mathrm{NiO}_{4}$ by $\mathrm{LSDA}+U$ method for (a) $\mathrm{Ni}^{2+}$ and (b) $\mathrm{Ni}^{3+}$. Energy zeroth is fixed at the top of the occupied bands. Inset shows the location of each site. Local magnetic moment of each site is $-1.46,-0.94,1.51$, $-1.51,0.93,1.46 \mu_{B}$, respectively, from the left-hand side to the right-hand side.

$\mathrm{NiO}_{6}$ octahedra is neglected hereafter. We, then, fix the total volume of unit cell and set the $c / a$ ratio to be the observed value 3.26. Crystallographic coordinates of atoms are fixed at those of $x=0$ and, with changing $x$, positions of all atoms are scaled. The magnetic unit cell at $x=0$ is a $\sqrt{2} \times \sqrt{2} \times 1$ supercell, and a $m \sqrt{2} \times \sqrt{2} \times 1$ supercell at $x=\frac{1}{m}$.

At $x=0$, calculated values of the band gap $(3.73 \mathrm{eV})$ and local magnetic moment $\left(1.54 \mu_{B}\right)$ agree well with the observed values of $4 \mathrm{eV}$ (Ref. 25) and $1.68 \mu_{B},{ }^{24}$ respectively. Figure 1 shows the projected density of states of LSNO at $x=\frac{1}{3}$. The position of this $x^{2}-y^{2}$ orbital on the $\mathrm{Ni}^{3+}$ site shifts to energy region just above the Fermi energy. The calculated energy gap is $0.10 \mathrm{eV}$. The resultant spin structure is such that antiferromagnetic domains are separated by hole stripe, and the magnetic moment localized on each site is -1.46 , $-0.94,1.51,-1.51,0.93,1.46 \mu_{B}$, respectively, from the lefthand side to the right-hand side. The center of the hole stripe is located on the $\mathrm{Ni}^{3+}$ ions, and no neighboring $\mathrm{Ni}^{3+}$ ion exists. The spins on two $\mathrm{Ni}^{2+}$ ions sharing the same neighboring $\mathrm{Ni}^{3+}$ ions are antiparallel to each other [inset of Fig. 1(a)]. Introduction of the multiple Slater determinant decreases the spin moments on $\mathrm{Ni}^{3+}$ ions, because the spin configuration with the opposite spin direction on $\mathrm{Ni}^{3+}$ ions gives the same energy with the present spin configuration. This order is consistent with the experimentally observed stripe and should be assigned to the real ground state. However, the calculated lowest energy state is of no charge order, and the energy is lower by $0.5 \mathrm{eV} /$ cell than that of the real ground state in Fig. 1. The spin-ordered alignment of this calculated lowest energy state is different from that of the charge ordered state shown in the inset of Fig. 1(a). The local magnetic moment of the calculated lowest energy state is, on each $\mathrm{Ni}$ site in Fig. 1(a), 1.48, 1.33, 1.48, -1.48, -1.33, $-1.48 \mu_{B}$, from the left-hand side to the right-hand side. 


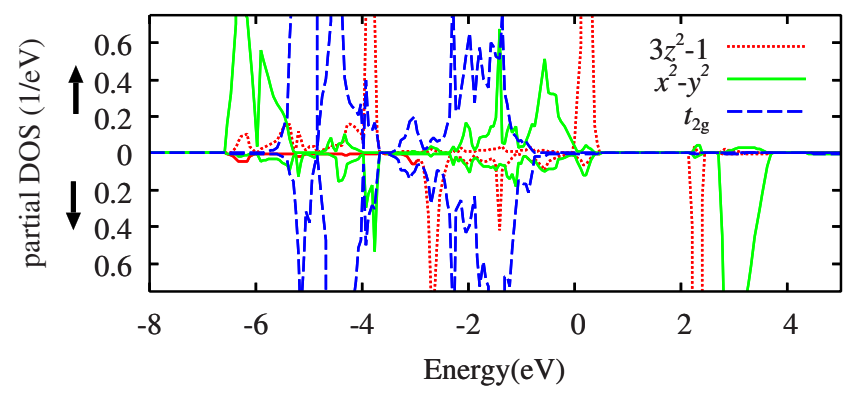

FIG. 2. (Color online) Projected density of states for each spin (up and down indicated by arrows) of electron in antiferromagnetic metallic LSNO $(x=1)$ by LSDA $+U$ method. Energy zeroth is fixed at the Fermi energy. Resultant electronic structure is antiferromagnetic metal (see text). The hole states are the $3 z^{2}-1$ orbital in contrast to the $x=\frac{1}{3}$ case. Localized magnetic moment on each Ni site is equal to $1.02 \mu_{B}$.

There are two problems responsible for the energy increase of the real ground state with the charge order. One is the absence of correlation energy arising from the charge fluctuation between $\mathrm{Ni}^{2+}$ and $\mathrm{Ni}^{3+}$ configuration. The other is the fact that the LSDA $+U$ method often underestimates the correlation energy of antiferromagnetic bonds.

\section{ELECTRONIC STRUCTURE OF LSNO $\left(x=\frac{1}{2}, 1\right)$ BY USING LSDA+ $U$ METHOD}

$\mathrm{LaSrNiO}_{4}(x=1)$ is observed to be paramagnetic metal, the occupation of the $\mathrm{Ni}$ ion distributes homogeneously and each ion is $\mathrm{Ni}^{3+}$. On the contrary, the calculated ground state is antiferromagnetic metal and the projected density of states is shown in Fig. 2. This contradiction between the observation and the calculation is due to unrealistic stabilization energy of the magnetically ordered state in LSDA $+U$ calculation of large on-site Coulomb interaction $U, J$, against the paramagnetic state. If we assume that the ground state is represented by multiple Slater determinants, the paramagnetic state would be represented as a linear combination of random spin configurations. Then spin polarization within atomic sphere lowers the on-site Coulomb and exchange interaction energy and correlation energy stabilize the paramagnetic metal phase. However, in the LSDA $+U$ method, the ground-state wave function is represented by a single Slater determinant. Consequently, the paramagnetic state is only possible in the state where all ions have no spin polarization. This increases the total energy much, coupling with large $U, J$. A dominant component of the real ground state at $x=1$ should be a linear combination of Slater determinants which have a single electron on each site and spins are not ordered.

The LSDA $+U$ calculation fails to present the paramagnetic metal phase as the ground state at $x=1$, but the correlation effects cause correct change of the splitting of two $e_{g}$ bands. Figure 2 shows that the $3 z^{2}-1$ and $x^{2}-y^{2}$ bands locate above and below the Fermi energy, respectively. Comparing Figs. 1 and 2, one can see the change of the hole characters from the case of $x=\frac{1}{3}$ to that of $x=\frac{1}{2}$. In the $x=\frac{1}{3}$ system, the hole is doped in the $x^{2}-y^{2}$ orbital, while it is doped in the $3 z^{2}-1$ orbital in the $x=1$ system. This change of the hole character agrees with the experimental results described in Sec. I. The origins of this change are the strong on-site Coulomb interaction $U$ between two orbitals and the difference of the dispersion of two orbitals or bands. In the LDA calculation of the system of $x=0$, two $e_{g}$ bands cross the Fermi energy. The $3 z^{2}-1$ band is narrow and lying on the energy region $-0.62 \mathrm{eV}-0.69 \mathrm{eV}$, and the $x^{2}-y^{2}$ band is wide and lying on the energy region $-0.86 \mathrm{eV}-2.29 \mathrm{eV}$, where the energy zeroth is fixed at the Fermi energy. When a small amount of hole is introduced, the hole is doped into the $x^{2}$ $-y^{2}$ band, because the $x^{2}-y^{2}$ band is averagely located higher than the $3 z^{2}-1$ band. At the hole concentration $x=1$, orbital polarization is maximized if only one band is occupied and the other is pushed up over the Fermi energy. Because the lowest energy levels of the $e_{g}$ band mainly consist of $x^{2}-y^{2}$ orbitals, the $x^{2}-y^{2}$ orbital is preferable to be occupied. Thus, the hole character changes from $x^{2}-y^{2}$ orbital to $3 z^{2}-1$ orbital at $x=1$. In LSDA $+U$ calculation, however, the spin and orbital polarization could not be completely maximized and the system becomes metal. The potential correction in Table I shows this relation between the hole character and the strong on-site Coulomb interaction $U$. Potential corrections are the derivatives of the Hubbard correction term of LSDA $+U$ Hamiltonian with respect to each element of local occupation matrix $\left\langle\hat{c}_{\alpha}^{\sigma^{\dagger}} \hat{c}_{\beta}^{\sigma}\right\rangle$ on each atom, where $\alpha$ and $\beta$ denote atomic orbitals and $\sigma$ spin. In the present system, potential corrections are diagonal matrices due to symmetry and only diagonal elements are listed in Table I. In such case, each diagonal element shows the energy level shift of the respective orbital induced by the on-site Coulomb interaction. Potential correction of $3 z^{2}-1 \uparrow\left(x=\frac{1}{3}\right)$ nearly equal to that of $x^{2}-y^{2} \uparrow(x=1)$ and that of $x^{2}-y^{2} \uparrow\left(x=\frac{1}{3}\right)$ nearly equal to that of $3 z^{2}-1 \uparrow(x=1)$. Therefore, the on-site Coulomb interaction applied to the narrow $3 z^{2}-1$ and wide $x^{2}-y^{2}$ band induces the difference of the hole character between the

TABLE I. Diagonal elements of potential correction (in units of eV). Potential correction is a derivative of the Hubbard correction term in LSDA $+U$ Hamiltonian with respect to elements of the occupation matrix. This gives the orbital-dependent potential corresponding to fluctuations of the orbital occupation.

\begin{tabular}{lcccc}
\hline \hline & $3 z^{2}-1 \uparrow$ & $x^{2}-y^{2} \uparrow$ & $3 z^{2}-1 \downarrow$ & $x^{2}-y^{2} \downarrow$ \\
\hline $\mathrm{Ni}^{2+}\left(x=\frac{1}{3}\right)$ & -2.07 & -2.06 & 3.80 & 3.29 \\
$\mathrm{Ni}^{3+}\left(x=\frac{1}{3}\right)$ & -1.61 & -0.18 & 2.92 & 2.10 \\
$\mathrm{Ni}^{3+}(x=1)$ & -0.01 & -1.67 & 2.56 & 2.92 \\
\hline \hline
\end{tabular}




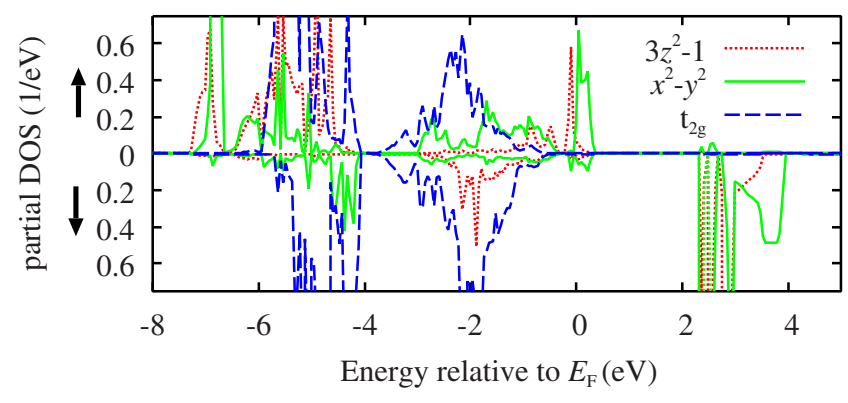

FIG. 3. (Color online) Projected density of states for each spin (up and down indicated by arrows) of electron in antiferromagnetic metallic LSNO $\left(x=\frac{1}{2}\right)$ by LSDA $+U$ method. Energy zeroth is fixed at the Fermi energy. Resultant electronic structure is antiferromagnetic metal (see text). Localized magnetic moment on each $\mathrm{Ni}$ site is equal to $\pm 1.3 \mu_{B}$.

low concentration $\left(x=\frac{1}{3}\right)$ case and the high concentration $(x$ =1) case.

In the high-doped $x=\frac{1}{2}$ case, the real system has a incommensurate stripe-type charge order, as described in Sec. I. The ground state of LSDA $+U$ calculation shows, however, no charge order. Consequently, the electronic structure of the calculated system is metallic as shown in Fig. 3.

The charge order of the system of $x=\frac{1}{2}$ would be expected to be a commensurate checkerboard-type order, because of the following three reasons. The first is that the periodic boundary condition forces the charge order to be commensurate to the lattice, and once we assume the incommensurability $\epsilon$ to be a rational value $\frac{1}{2}$, the resultant charge order is checkerboard type. The second is that in the temperature range $T_{\mathrm{SO}}<T<T_{\mathrm{CO}}$, the checker-board type charge order is observed in the real system with $x=\frac{1}{2}$. The third reason is that the positive value of intersite Coulomb interaction stabilizes the checkerboard-type charge order. We must notice that the intersite Coulomb interaction is included in LSDA $+U$ Hamiltonian by means of Hartree energy.

We could not find a converged charge ordered solution, even as a metastable state, by using LSDA $+U$ calculation. Localized magnetic moments of the four $\mathrm{Ni}$ ions in the unit cell are equal to $1.29,1.32,-1.29,-1.32 \mu_{B}$, respectively. Therefore, all the Ni ions are of the same type, allowing the difference of local magnetic moment by $3 \%$. Figure 3 shows the partial density of state for each orbital of $\mathrm{Ni}$ ions.

The Hartree energy may be insufficient to stabilize the charge order. In the real system, the electron localized on the $\mathrm{Ni}^{2+}$ site has an extending tail on the $\mathrm{Ni}^{3+}$ site and the center of the localized electron can be fluctuating among $\mathrm{Ni}^{2+}$ and surrounding $\mathrm{Ni}^{3+}$ sites, in order to lower the correlation energy. Because the ground-state wave function is represented by a single Slater determinant in the LSDA $+U$ calculation, the fluctuation of the center of the localized electron is neglected, then the correlation energy is underestimated. Moreover, in the Hartree energy, the contribution of the tail to the charge on the $\mathrm{Ni}^{3+}$ site and the contribution of the head to the charge on the $\mathrm{Ni}^{2+}$ site are treated as if they were separate electrons. Such treatment gives an additional self-interaction and causes the increase of the estimated energy. Because the number of nearest neighbor (NN) pairs of $\mathrm{Ni}^{2+}$ and $\mathrm{Ni}^{3+}$ ions are maximized in the checkerboard-type charge ordered system, the above two reasons become serious for the increase of estimated energy.

Now the prescription for resolving these problems is the following. We need to preserve the antisymmetry for exchanging of any pair of single electron wave functions in order to remove self-interaction, we need to employ as many configurations of Slater determinants as possible in order to correctly estimate the correlation energy. Thus, we employ the exact diagonalization of the many-body Hamiltonian in the following sections.

\section{DOUBLE ORBITAL EXTENDED HUBBARD HAMILTONIAN FOR LSNO $\left(x=\frac{1}{2}\right)$ BASED ON LDA CALCULATION}

To understand the ground state at $x=\frac{1}{2}$, we adopt the extended Hubbard Hamiltonian $\hat{H}$ of $e_{g}$ electrons on a twodimensional (2D) simple square lattice derived from results of LSDA $+U$ calculations, and diagonalize this many-body Hamiltonian exactly by using the Lanczos method with the inverse-iteration method,

$$
\begin{aligned}
\hat{H}= & \sum_{i, j, \alpha, \beta, \sigma} t_{i \alpha j \beta} \hat{c}_{i \alpha \sigma}^{\dagger} \hat{c}_{j \beta \sigma}+\sum_{i, \alpha, \sigma} \varepsilon_{i \alpha} \hat{c}_{i \alpha \sigma}^{\dagger} \hat{c}_{i \alpha \sigma} \\
& +\frac{1}{2} \sum_{\substack{i, \alpha, \beta, \gamma, \delta, \sigma, \sigma^{\prime}}} U_{\alpha \beta \gamma \delta} \hat{c}_{i \alpha \sigma^{\prime}}^{\dagger} \hat{c}_{i \beta \sigma^{\prime}}^{\dagger} \hat{c}_{i \delta \sigma^{\prime}} \hat{c}_{i \gamma \sigma} \\
& +\frac{V}{2} \sum_{\langle i, j\rangle, \alpha,} \hat{c}_{i \alpha \sigma}^{\dagger} \hat{c}_{i \alpha \sigma} \hat{c}_{j \beta \sigma^{\prime}}^{\dagger} \hat{c}_{j \beta \sigma^{\prime}}, \\
\beta, \sigma, \sigma^{\prime} &
\end{aligned}
$$

where the braces $\langle\cdots\rangle$ denote the summation over nearestneighbor (NN) pairs and the symbol $t$ denotes the SlaterKoster-type hopping parameters. The on-site energy $\varepsilon_{i \alpha}$ 's are determined by LDA calculation at $x=0:{ }^{26} t_{d d \sigma}=-0.543 \mathrm{eV}$, $t_{d d \delta}=0.058 \mathrm{eV}$ for the $\mathrm{NN}$ pairs and $\frac{1}{4} t_{d d \sigma}^{\prime}+\frac{3}{4} t_{d d \delta}^{\prime}=$ $-0.018 \mathrm{eV}, t_{d d \pi}^{\prime}=-0.023 \mathrm{eV}$ for the second $\mathrm{NN}$ pairs and $\Delta$ $=\varepsilon_{3 z^{2}-1}-\varepsilon_{x^{2}-y^{2}}=0.97 \mathrm{eV}$. The energy zeroth is set at the middle of $\varepsilon_{3 z^{2}-1}$ and $\varepsilon_{x^{2}-y^{2}}$. The matrix elements of the intraatomic Coulomb interactions $U_{\alpha \beta \gamma \delta}$ are represented as functions of averaged Coulomb and exchange interations $U$ and $J$, by using the same expressions as in LSDA $+U$ method. ${ }^{18}$ The values of $U, J$ are chosen to be $7.5 \mathrm{eV}, 0.88 \mathrm{eV}$, respectively, on all sites. The value of the intersite Coulomb interaction $V$ is chosen as $0.5 \mathrm{eV}$, except explicit indication of the value of $V$. Later we will explain the reason of this choice, $V=0.5 \mathrm{eV}$, in detail.

Because two diagonal directions are inequivalent in the real LSNO $\left(x=\frac{1}{2}\right)$, we introduce a parameter $\delta$ showing the anisotropy of the second $\mathrm{NN}$ (diagonal) hopping parameter $t^{\prime}$. We add to or subtract from $t_{x^{2}-y^{2}, x^{2}-y^{2}}^{\prime}$ as $t_{x^{2}-y^{2}, x^{2}-y^{2}}^{\prime} \pm \delta$, depending on the direction, as depicted in Fig. 4(c). We investigate the $\delta$ dependence in the range of $0 \leqslant \delta \leqslant 0.02$ so that the sign of the second NN hopping parameter does not change. This anisotropy $\delta$ reserves translational symmetry. 


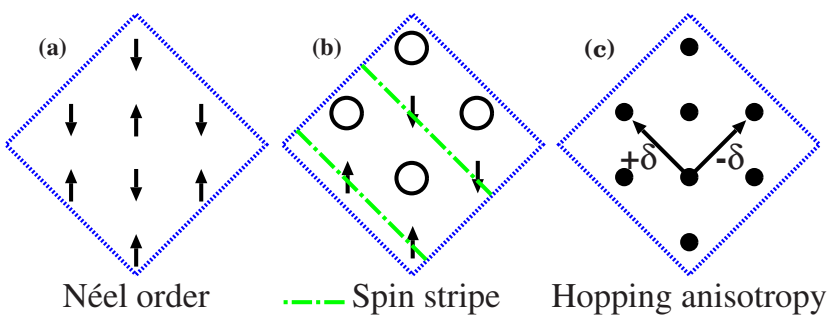

FIG. 4. (Color online) Two spin configurations at $x=\frac{1}{2}$ in a $\sqrt{8}$ $\times \sqrt{8}$ cell: (a) Néel order and (b) one of charge and spin stripe order among doubly degenerate ones. Up and down arrows in (a) and (b) denote spins and circles in (b) denote holes. In Néel order, spin correlation of four nearest neighbors has negative sign. Those of two second nearest neighbors and a third nearest neighbor have positive sign. In stripe order, two second nearest neighbors have opposite signs with each other. (c) The hopping anisotropy of second nearest neighbors.

As a result, the finite value of $\delta$ does not induce charge order, at least in the present range of $\delta$. For example, the difference between the charge-charge correlation of the ground state at $V=0 \mathrm{eV}$ under $\delta=0.02$ and $\delta=0$ is at most $3 \%$. However, once charge order is induced by other quantities (in the present case, by intersite Coulomb interaction $V$ ), finite value of $\delta$ changes essentially the spin order of the system, as is shown in Sec. VII. Finite value of $\delta$ neither changes essentially excitation spectra and energy gap. The width of energy gap reduced by a few percent at $V=0.5 \mathrm{eV}$. Therefore, we fix the value of $\delta$ at $\delta=0$ in Secs. IV-VI. We will change the value of $\delta$ in Sec. VII, where the change of spin order induced by $\delta$ is discussed.

We diagonalize the many-body Hamiltonian of a system of 12 electrons on the planar $\sqrt{8} \times \sqrt{8}$ supercell, where each site has two $e_{g}$ orbitals. A periodic boundary condition is imposed, avoiding the bunching of electron at corners, and causes commensurate checkerboard-type order in the exact diagonalization result instead of incommensurate charge stripe in real LSNO $\left(x=\frac{1}{2}\right)$ as is mentioned in Sec. III. The total $\mathbf{S}^{2}$ and $S_{z}$ of this system is invariant, due to spherical symmetry of the spin space. We then restrict the Hilbert space so as to total $S_{z}=0$, which reduces the dimension of Hamiltonian to $\left({ }^{16} \mathrm{C}_{6}\right)^{2}=64,128,064$.

\section{CHARGE ORDER OF LSNO $\left(x=\frac{1}{2}\right)$}

At first, we discuss the ground state of LSNO at $x=\frac{1}{2}$. With increasing $V$, the ground state changes from singly degenerate state (state $S$ ) in the region $0 \mathrm{eV}<V<0.41 \mathrm{eV}$ to doubly degenerate (state $D$ ) $0.41 \mathrm{eV}<V<1 \mathrm{eV}$. Smoothness of the connection among respective series of states $S$ and $D$ with respect to $V$ is discussed in Appendix A.

Figure 5 shows the $V$ dependence of the total energies and the correlation functions of charge fluctuation $\left\langle\delta \hat{n}_{i} \delta \hat{n}_{j}\right\rangle$, where $\delta \hat{n}_{i}=\hat{n}_{i}-\left\langle\hat{n}_{i}\right\rangle$ and $\delta$ is fixed at 0 . At $V=0.5 \mathrm{eV}$, charge correlations $\left\langle\delta \hat{n}_{i} \delta \hat{n}_{j}\right\rangle$ of state $D$ equal to $-0.135,0.093,0.096$ for $\mathrm{NN}[(i, j)=(1,2)]$, second $\mathrm{NN}[(i, j)=(1,3)]$, and third $\mathrm{NN}[(i, j)=(1,7)]$ pairs, respectively, and this corresponds to

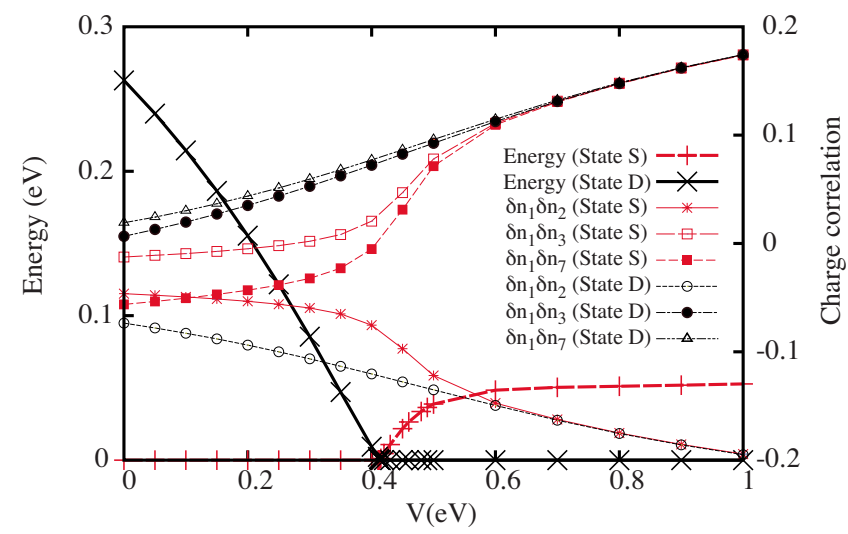

FIG. 5. (Color online) $V$ dependence of total energies and charge-charge correlations at $x=\frac{1}{2}$ and $\delta=0$, where $V$ is intersite Coulomb interaction. The state labeled " $S$ " is singly degenerate while the other state labeled " $D$ " is doubly degenerate. Energy zeroth is set at the ground-state energy. State $S$ is a ground state on the region $0 \mathrm{eV}<V<0.41 \mathrm{eV}$, state $D$ is a ground state on the region $0.41 \mathrm{eV}<V<1 \mathrm{eV}$. Suffixes 1, 2, 3, 7 denote the following sites: origin, nearest neighbor, second nearest neighbor, and third nearest neighbor, respectively. These site indexes are depicted in Fig. 6(a).

checkerboard-type charge order shown in Fig. 6(b). The checkerboard-type charge order in state $D$ exists even at $V$ $=0 \mathrm{eV}$, though the amplitude is very small. The charge correlations for $\mathrm{NN}$, second $\mathrm{NN}$, and third $\mathrm{NN}$ pairs at $V$ $=0 \mathrm{eV}$ are equal to $-0.074,0.007,0.019$, respectively. These values satisfy the checkerboard-type charge order: The positive correlation between the second NN pair is nearly equal to that between the third NN pair, and the correlation between the first NN pair is negative. The charge correlations in state $S$ are homogeneous in comparison with those in state $D$. The charge correlation in state $S$ at $V=0 \mathrm{eV}$ of first, second, and third NN pairs is equal to $-0.046,-0.013,-0.056$, respectively. Therefore, state $S$ at $V=0 \mathrm{eV}$ does not have any charge order. The absence of charge order in state $S$ in $0 \mathrm{eV}<V<0.4 \mathrm{eV}$ is attributed to a slow increase of charge correlations with increasing $V$ in this region of $V$.

Now we discuss three features in Fig. 5. Two are about correlation functions of state $S$ and one is about the energy difference between states $S$ and $D$; (i) rapid increase of the
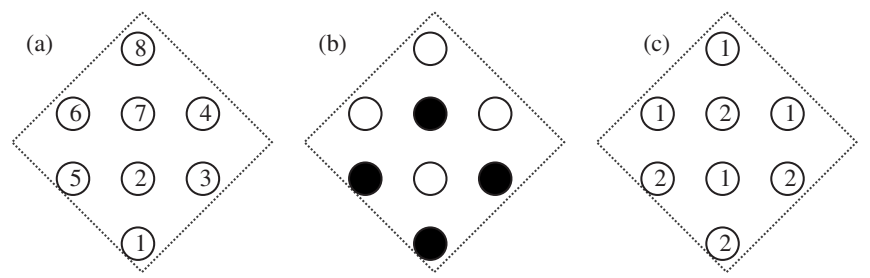

FIG. 6. (a) Site index of the present system at $x=\frac{1}{2}$ in a $\sqrt{8}$ $\times \sqrt{8}$ cell. (b) A type of hole distribution of the Slater determinants with the complete checkerboard-type charge order (SDCCO in short), where closed and open circle denote nonhole and hole $\mathrm{Ni}$ site, respectively. Another type of distribution is obtained by exchanging the hole site and nonhole site. (c) The occupation distribution of SDCCO in (b). A number in the open circle shows the occupation number of the respective site. 


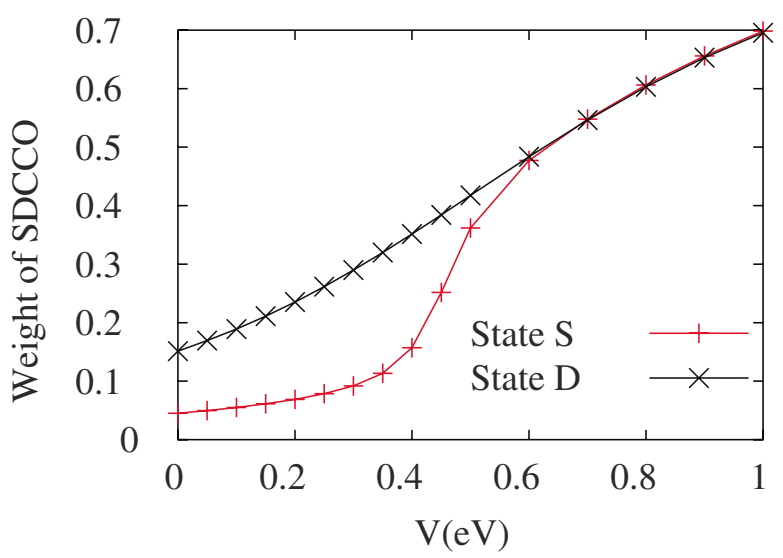

FIG. 7. (Color online) $V$ dependence of the weight of Slater determinants with complete checkerboard-type charge order (SDCCO in short), where $V$ is intersite Coulomb interaction. The states labeled " $S$ " and " $D$ " are the same states as in Fig. 5. $\delta=0$.

charge correlation of state $S$ in the region $0.4 \mathrm{eV}<V$ $<0.6 \mathrm{eV}$, (ii) merging charge correlation of state $S$ into that of state $D$, in the region $V>0.6 \mathrm{eV}$, and (iii) the constant energy difference between states $S$ and $D$ in the region $V$ $>0.6 \mathrm{eV}$. All these features are related to respective wave functions of states $S$ and $D$.

Let us define the ideal complete checkerboard-type charge order whose Slater determinant wave function (SDCCO) should satisfy the following two conditions, (a) and (b), then we specify the characteristics of real wave functions of states $S$ and $D$ by using the overlap with SDCCO's: (a) every site has occupation one or two, and (b) all neighboring sites of a singly occupied site are doubly occupied sites and vice versa. A type of their distribution of occupation is shown in Fig. 6(c). Assuming both transfer integral and on-site exchange parameter $J$ equal to zero, SDCCO's would be the ground states, where all the spin configurations are degenerate, because the numbers of neighboring hole $\left(\mathrm{Ni}^{3+}\right)$ and nonhole $\left(\mathrm{Ni}^{2+}\right)$ pairs are maximized. In an actual calculation, transfer integrals are finite and the SDCCO's are not eigenstate. The value of $V$ determines how much SDCCO's are hybridized into respective eigenstates. The more $V$ increases, the more hybridization of SDCCO's are preferable energetically.

In the region of $0 \mathrm{eV}<V<0.4 \mathrm{eV}$, the ground state is homogeneously extending state $S$ since the kinetic energy is a source of gain of the total energy. On the other hand, in the region of $0.4 \mathrm{eV}<V$, the ground state changes to the charge ordered state $D$ since the source of energy gain is the correlation energy due to the intersite Coulomb interaction $V$. The dependence of the total weight of SDCCO's is shown in Fig. 7. Comparing Figs. 5 and 7, the above features (i), (ii), and (iii) appear as follows. Since the wave function of the ground state $S$ is homogeneous, the overlap with SDCCO is small in the region of $0 \mathrm{eV}<V<0.4 \mathrm{eV}$. We discuss more details about the weight of SDCCO's of state $S$ at $V=0 \mathrm{eV}$ in Appendix $\mathrm{B}$. Then, in the range $0.4 \mathrm{eV}<V<0.6 \mathrm{eV}$, the weight of SDCCO's rapidly increases. Because both states $S$ and $D$ are eigenstates of $\hat{H}$ and orthogonal with each other, the co-

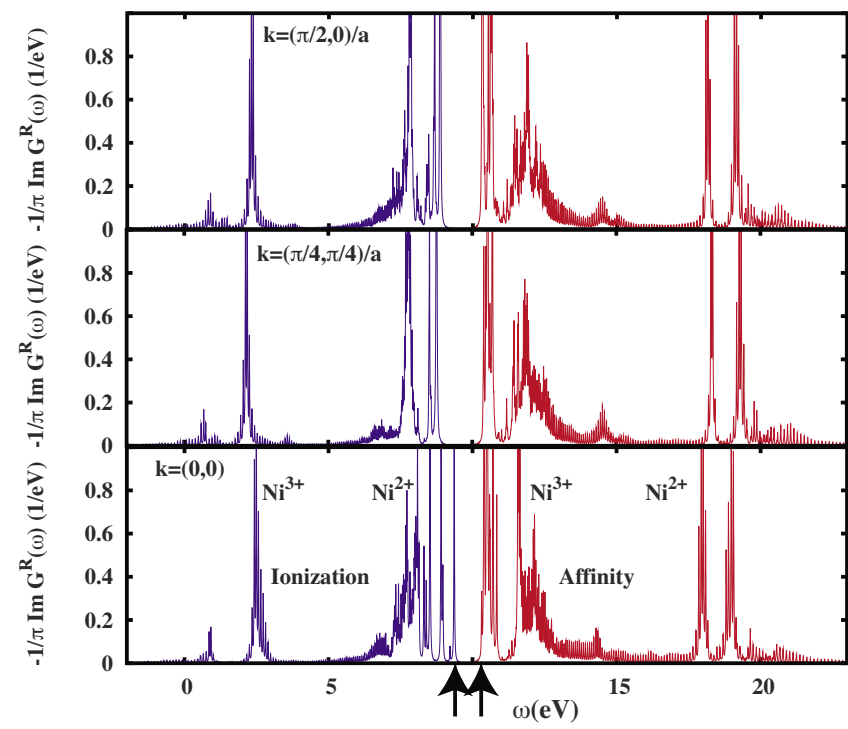

FIG. 8. (Color online) Single-particle spectral function of eight sites, $N=12\left(x=\frac{1}{2}\right)$ system with $\delta=0$ and $V=0.5 \mathrm{eV}$. Three panels are corresponding to respective $\boldsymbol{k}$ points, $\left(\frac{\pi}{2}, 0\right) \frac{1}{a},\left(\frac{\pi}{4}, \frac{\pi}{4}\right) \frac{1}{a},(0,0)$, from the top to the bottom. Each value of $\boldsymbol{k}$ corresponds to a different boundary condition. Two arrows near $10 \mathrm{eV}$ shows the position of the highest ionization level $9.4 \mathrm{eV}$ and the lowest affinity level $10.3 \mathrm{eV}$ in the case of $\boldsymbol{k}=(0,0) \frac{1}{a}$. Spectral functions are approximated by the polynomial of 800 degrees in $\hat{H}$, where $\hat{H}$ denotes the Hamiltonian. A small imaginary part $(0.01 \mathrm{eV})$ is added to the energy $\omega$ for smearing $\delta$-function peaks.

efficients of SDCCO's in state $S$ are different from those in state $D$. In the range $0.4 \mathrm{eV}<V<1 \mathrm{eV}$, states $S$ and $D$ have the same charge order but the spin order is quite different, which is discussed in Sec. VII. Then, the difference of the total energies between states $S$ and $D$ is attributed to the difference of spin configurations. As is discussed in Sec. I, $T_{\mathrm{SO}}<T_{\mathrm{CO}}$ in the real system with $x=\frac{1}{2}$ and, therefore, the energy scale of spin order is smaller than that of charge order. In fact, we can see a large energy difference between states $S$ and $D$ in the region of $0 \mathrm{eV}<V<0.4 \mathrm{eV}$, since the charge order is different between states $S$ and $D$ here. Then, the characteristics of the charge order are the same in states $S$ and $D$ in the range $0.6 \mathrm{eV}<V<1 \mathrm{eV}$ [feature (ii)], as seen in the charge correlation functions and the same weights of SDCCO's in states $S$ and $D$, and the constant energy difference between states $S$ and $D$ [feature (iii)]. The observed high-temperature state $\left(T>T_{\mathrm{SO}}\right)$ in LSNO of $x=\frac{1}{2}$ might be a mixture of charge ordered eigenstates with significant weight of SDCCO's, including the doubly degenerate states $D$ and the singly degenerate state $S$.

As mentioned in Sec. I, the real ground state of LSNO $\left(x=\frac{1}{2}\right)$ shows charge stripe order, incommensurate to the lattice. And as mentioned in Sec. III, the periodic boundary condition forces the charge order to be commensurate charge order of checkerboard type. Therefore, the ground state of the Hamiltonian should have checkerboard-type charge order, and we choose $V=0.5 \mathrm{eV}$ from now on. The value 
$V=0.5 \mathrm{eV}$ is not very unrealistic because a recent paper shows $V=0.34 \mathrm{eV}$ in the GW approximation of $\mathrm{LaMnO}_{3}{ }^{27}$

\section{EXCITATION SPECTRA AND ENERGY GAP OF LSNO $\left(X=\frac{1}{2}\right)$}

We show calculated single-particle spectral functions of the system with $x=\frac{1}{2}$ in Fig. 8, in order to see whether the spectra have energy gap and the system is insulator as in the observed case. Intersite Coulomb interaction is fixed at $V$ $=0.5 \mathrm{eV}$ as discussed in Sec. V. Three $\boldsymbol{k}$ points are chosen; $\boldsymbol{k}=(0,0) \frac{1}{a},\left(\frac{\pi}{4}, \frac{\pi}{4}\right) \frac{1}{a}$, and $\left(\frac{\pi}{2}, 0\right) \frac{1}{a}$, where $a$ is the nearest Ni-Ni distance, the positions of $\mathrm{Ni}$ ions are $(n, m) a$, and the two translation vectors are $(-2,2) a$ and $(2,2) a$. Each $\boldsymbol{k}$ point corresponds to periodic or antiperiodic boundary condition along respective translation vector. In order to obtain continuous spectra, we introduce an imaginary energy $\eta$ of $0.01 \mathrm{eV}$ and the value of $\eta$ determines the resolution of the spectra. The value of $\eta$ must satisfy $\eta \geq \frac{\text { total width of spectra }}{m}$, where $m$ denotes the the highest power of $\hat{H}$, because $m$ is the upper limit of the number of $\delta$ functions in the spectra. In the Lanczos method, the truncation error easily breaks down the orthogonality of the Lanczos vectors. Because calculating the spectral function may need the large $m$, we employ the shifted-COCG method, ${ }^{28}$ which is a variant of the conjugate gradient $(\mathrm{CG})$ method. It must be noticed that the shifted-COCG method is numerically stable. Because shifted-COCG method requires a reference energy in the energy region of a peak of spectral weight, we need to know a rough profile of the spectral weight. Therefore, the shiftedCOCG method should be used only when we need a very fine profile of the spectral function. More details will be explained elsewhere.

In the present case, the "total width of spectra" is roughly estimated as $E\left(\mathrm{Ni}^{2+}\right)-E\left(\mathrm{Ni}^{3+}\right) \sim U=7.5 \mathrm{eV}$ for ionization levels, and affinity levels, separately. Then we choose $m$ $=800$. We calculate the spectral function of $m=160$ by the Lanczos method, and then calculate that of $m=800$ by the shifted-COCG method. Actually, the width of spectra is wider than $U$ due to the mixing of higher or lower occupation configurations. The spiky structure of spectra is an artifact due to the choice of smaller $\eta=0.01 \mathrm{eV}$. Our choice of the value of $\eta$ is intended to show the gap structure between ionization levels and affinity levels, at every $\boldsymbol{k}$ point. We fix the value of hopping anisotropy $\delta=0$. The hopping anisotropy $\delta$ does not change the shape of the spectral function in the range of $0 \leqslant \delta<0.02$. The width of the gap at $k$ $=(0,0) \frac{1}{a}$ in Fig. 8 is smallest among three $\boldsymbol{k}$ points, where the highest ionization level (HIL) is located at $9.4 \mathrm{eV}$ and the lowest affinity level (LAL) is at $10.3 \mathrm{eV}$. Therefore, we conclude that this system is an insulator with an energy gap of $0.9 \mathrm{eV}$.

It is important to know the symmetry of the single electron wave functions of HIL and LAL, in order to show that the "gap" is not an artifact of discretized $\boldsymbol{k}$ point. The symmetry of the respective single electron wave function can be labeled by crystal momentum $\boldsymbol{k}$. If the "gap" is an artifact and the ground state is metal, HIL and LAL are labeled by different $\boldsymbol{k}$. Actually many levels overlap and form a single peaklike structure because of complicated interactions, especially in the energy range near LAL. Even the definition of the "single electron wave function" is not clear in such a circumstance. Therefore, we define a "single electron wave function" as follows here. First we choose the bottom $\left(E_{b}\right)$ and top $\left(E_{t}\right)$ of a single peaklike structure. Next we integrate the Green function of the matrix form in the energy range $E_{b}<E<E_{t}$,

$$
-\frac{1}{\pi} \int_{E_{b}}^{E_{t}} \operatorname{Im} G_{i \alpha j \beta}^{R}(\omega) d \omega .
$$

and then we diagonalize it. This matrix corresponds to energetically partitioned $\left\langle\hat{c}_{i \alpha}^{\dagger} \hat{c}_{j \beta}\right\rangle\left(E_{b}<E_{t}<E_{F}\right)$ or $\left\langle\hat{c}_{i \alpha} \hat{c}_{j \beta}^{\dagger}\right\rangle\left(E_{F}\right.$ $\left.<E_{b}<E_{t}\right)$. Therefore, the eigenvector of the above matrix corresponds to the single electron wave function related to the peaklike structure, and the eigenvalue corresponds to the occupation number of the single electron wave function. We summarize in Table II the $E_{b}, E_{t}$, eigenvalues and the $\vec{k}$ representing the symmetry of eigenvectors for the peaklike structure near HIL and LAL. At $\boldsymbol{k}=(0,0) \frac{1}{a}$, HIL and LAL single electron wave functions share the same symmetry. This cannot occur in the metallic system. Therefore, we can conclude that the split between HIL and LAL is not an artifact and the system is an insulator.

Now we show that the intersite Coulomb interaction $V$ induces the energy gap between occupied and unoccupied states, not on-site Coulomb interaction $U$. Figures 9(a) and 9(b) show the "spectral function" with respect to state $D$ with $V=0.5,0.2$, and $0 \mathrm{eV}$. Note that, since state $D$ is not the ground state at $V=0.2 \mathrm{eV}$ and $V=0 \mathrm{eV}$, they (for $V$ $=0.2 \mathrm{eV}$ and $V=0.5 \mathrm{eV}$ cases) are not satisfying the definition of the spectral function. These spectral functions are approximated by rational function of the degree of $\hat{H}^{160}$ by using the Lanczos method. The top panel of Fig. 9(a) and the bottom panel of Fig. 8 are the same, except that the latter uses a higher degree of $\hat{H}^{800}$ and the shifted COCG. They are sharing characteristic peaks of the HIL and LAL. Therefore, 160 degrees is enough to discuss the characteristics of spectra. At $V=0.5 \mathrm{eV}$ (top panel), HIL and LAL are $\left(x^{2}-y^{2}\right)$ and $\left(3 z^{2}-1\right)$ orbitals, respectively, and this agrees well with experimental observation and the spectrum in Fig. 3. At $V$ $=0.2 \mathrm{eV}$ (middle panel), HIL lowers to $7.9 \mathrm{eV}$ and the energy gap becomes smaller than that at $V=0.5 \mathrm{eV}$. At $V$ $=0 \mathrm{eV}$ (bottom panel), HIL lowers to $6.9 \mathrm{eV}$ and two excitation peaks overlap with each other in HIL and LAL, i.e., the energy gap vanishes. Thus, we can conclude that the system with $x=\frac{1}{2}$ becomes the insulator due to $V$, not $U$, and the intersite Coulomb interaction $V$ causes the energy stabilization and the opening of the gap of the ground state $D$. The gap width in the bulk limit is discussed in Appendix C.

\section{SPIN ORDER OF LSNO $\left(x=\frac{1}{2}\right)$}

We discuss the spin stripe order induced by the anisotropy of hopping integral between the $\mathrm{NN}$ sites. Introduction of the anisotropy does not contradict the local symmetry of the ob- 
TABLE II. Bottom and top of the energy of the peaklike structure, area of the structure, the largest eigenvalue (and its degeneracy after " $\times$ " symbol) related to "single electron wave function" (see text) and the representative $\vec{k}$, which shows the symmetry of the single electron wave function. In the right-hand column, single $\vec{k}$ is written and the other equivalent $(\vec{k})$ are omitted. HIL and LAL stand for highest ionization level (HIL) and lowest affinity level (LAL), respectively. Each $\boldsymbol{k}$ point corresponding to the boundary conditions is written in the leading lines. $\delta=0$.

\begin{tabular}{|c|c|c|c|c|c|}
\hline & $E_{b}(\mathrm{eV})$ & $E_{t}(\mathrm{eV})$ & $\begin{array}{c}\text { Peak } \\
\text { area }\end{array}$ & $\begin{array}{l}\text { The largest } \\
\text { eigenvalue and } \\
\text { its degeneracy }\end{array}$ & $\begin{array}{c}\text { Symmetry of } \\
\text { eigenvectors } \\
\vec{k}\left(\frac{1}{a}\right)\end{array}$ \\
\hline \multicolumn{6}{|c|}{ At $\vec{k}=(0,0) \frac{1}{a}$} \\
\hline HIL & 9.15 & 9.55 & 0.37 & $0.09 \times 4$ & $\left(\frac{\pi}{2}, \frac{\pi}{2}\right)$ \\
\hline LAL & 10.05 & 11.15 & 2.87 & $0.41 \times 4$ & $\left(\frac{\pi}{2}, \frac{\pi}{2}\right)$ \\
\hline \multicolumn{6}{|c|}{ At $\vec{k}=\left(\frac{\pi}{4}, \frac{\pi}{4}\right) \frac{1}{a}$} \\
\hline HIL & 8.18 & 8.98 & 1.72 & $0.63 \times 2$ & $\left(\frac{\pi}{4}, \frac{\pi}{4}\right)$ \\
\hline LAL & 10.02 & 11.02 & 2.99 & $0.44 \times 4$ & $\left(\frac{-\pi}{4}, \frac{3 \pi}{4}\right)$ \\
\hline \multicolumn{6}{|c|}{ At $\vec{k}=\left(\frac{\pi}{2}, 0\right) \frac{1}{a}$} \\
\hline HIL & 8.18 & 9.18 & 1.88 & $0.38 \times 4$ & $\left(\frac{\pi}{2}, 0\right)$ \\
\hline LAL & 10.12 & 11.12 & 2.90 & $0.42 \times 4$ & $\left(\frac{\pi}{2}, \pi\right)$ \\
\hline
\end{tabular}

served charge and spin stripe. This anisotropy with $\delta$ may couple with orthorhombic lattice distortion of $T_{2 g}$ symmetry. Coupling to the distortion reminds us of the JT mechanism. However, the $T_{2 g}$ distortion is not a JT mode for the $e_{g}$ electron, and therefore, this mechanism is not of the JT mechanism.

The spin correlation function $\left\langle\mathbf{S}_{i} \cdot \mathbf{S}_{j}\right\rangle$ at $\delta=0$ is equal to $-0.37,0.12,-0.07$, respectively, when $V=0.5 \mathrm{eV}$ and shows a lack of long range order. Equivalence of two diagonal directions is an origin of the twofold degeneracy, as is shown in the result of $\boldsymbol{k}=\left(\frac{\pi}{4}, \frac{\pi}{4}\right) \frac{1}{a}$ in Sec. V.

Because a large number of holes are doped in the $x^{2}-y^{2}$ orbital, the electron in the $x^{2}-y^{2}$ orbital on $\mathrm{Ni}^{2+}$ can have a long tail extending to the $\mathrm{Ni}^{3+}$ site. The hopping part of the total energy per electron is $-0.30 \mathrm{eV}$, and its absolute value is 2 times as large as that in the parent material $(x=0, N$ $=16),-0.15 \mathrm{eV}$. Following the localized electron picture, an increase of hopping energy indicates larger hybridization of $x^{2}-y^{2}$ orbital between $\mathrm{Ni}^{2+}$ and $\mathrm{Ni}^{3+}$. Then, spin on the $\mathrm{Ni}^{2+}$ site has strong correlation with that on the $\mathrm{Ni}^{3+}$ site. Thus, the spin correlation in the $x=\frac{1}{2}$ system is more complicated than that in the $x=0$ system where the localized spin picture is applicable.

The present parameter set in $\hat{H}$ is another origin of the complexity of spin structure. If $J \gg \Delta$, the majority spin is preferable in order to lower the exchange energy when the electron visited the $\mathrm{Ni}^{3+}$ site. In contrast, if $\Delta \gg J$, the minority spin is preferable in order to lower on-site energy. The present values of $J$ and $\Delta$ satisfy neither of the two conditions. In addition to that, transfer integral and intersite Coulomb interaction $V$ has the same energy scale. This causes the competition between the extended state and the localized single electron wave function. All these competitions may cause the instability against the perturbation.

In the case of $\delta \neq 0$, the ground-state energy decreases with increasing $\delta$ and can be expressed as $\left(36.75-104.3 \delta^{2}\right) \mathrm{eV}$. The spin correlation function is depicted in Fig. 10. The value of the correlation function between nearest neighbors, $\left\langle\mathbf{S}_{1} \cdot \mathbf{S}_{2}\right\rangle=-0.37--0.38$, does not change. The local spin moment is $\left\langle\mathbf{S}_{i}^{2}\right\rangle=1.31$. If two electrons are localized on one site in a triplet state, four electrons are localized on the four NN sites, and the total spin $S$ of this totally six-electron system is equal to 1 , then $\left\langle\mathbf{S}_{1} \cdot \mathbf{S}_{2}\right\rangle=$ -0.375 , which is almost the same as the calculated value of $\left\langle\mathbf{S}_{1} \cdot \mathbf{S}_{2}\right\rangle$. In the range of $\delta \geq 0.01 \mathrm{eV}$, correlation functions of the two types of second neighbor pairs have opposite signs and the structure of the calculated spin stripe order is consistent with the observed one. This critical value $\delta=0.01 \mathrm{eV}$ is less than $2 \%$ of the absolute value of the $\mathrm{NN}$ hopping $t_{d d \sigma}$, and thus small anisotropy changes the spin structure drastically.

The spin order is quite different between the systems of the single-orbital and of the multiorbitals. The former on the 2D square lattice is the Néel order on the $\sqrt{2} \times \sqrt{2}$ cell, ${ }^{15}$ and the latter is the charge and spin stripe. Once we assume only $x^{2}-y^{2}$ orbital at each site and set eight electrons on 16 sites, we get the value of $\left\langle\mathbf{S}_{1} \cdot \mathbf{S}_{2}\right\rangle$ equal to -0.03 , which is very small compared to the value -0.38 in LSNO. ${ }^{30}$ This reduction of $\left\langle\mathbf{S}_{1} \cdot \mathbf{S}_{2}\right\rangle$ in the single-orbital system is attributed to the absence of localized spin on a hole site and consistent with the Néel order mentioned above. However, a spin remains on a hole site in LSNO $\left(x=\frac{1}{2}\right)$. Since two neighboring sites of a hole site have spins of opposite direction with each other, the spin state of a hole site is a linear combination of up and down spin due to symmetry. Because no term in the Hamiltonian flips a spin on a hole site, two antiparallel-spin electrons on two hole sites must exchange their positions through the hopping process, in order to create such a linear combination. This hopping process lowers correlation energy, and the ground state must be represented by the multiple Slater determinant. In all hopping processes to create the multiple Slater determinants, that of exchanging a hole and a nonhole 

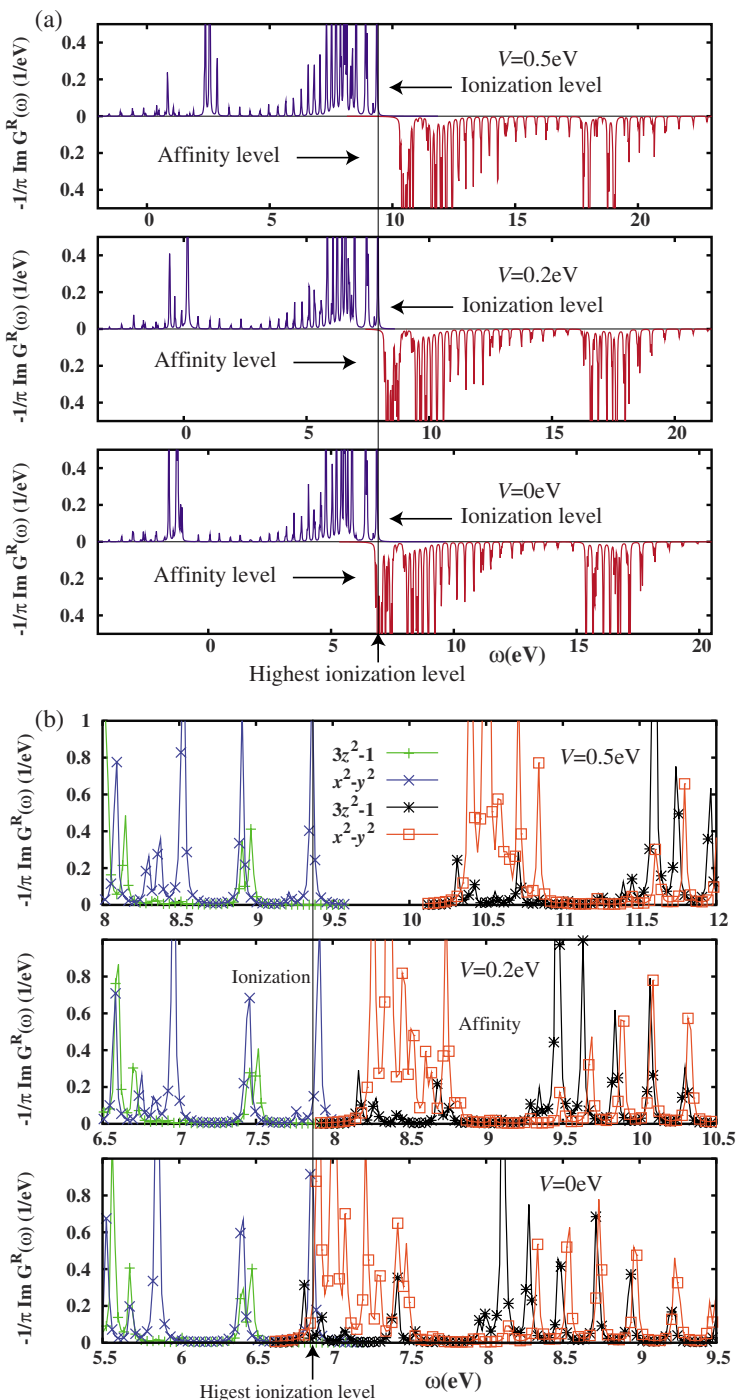

FIG. 9. (Color online) Spectral functions of eight sites, $N=12$ $\left(x=\frac{1}{2}\right)$ system are drawn at $\delta=0$. (a) Each panel corresponds to $V$ $=0.5,0.2$, and $0 \mathrm{eV}$ from the top to the bottom. In order to show the overlap between the ionization levels and the affinity levels at $V$ $=0 \mathrm{eV}$, they are drawn separately in the upper and the lower halves of respective panels. Spectral functions are approximated by the polynomial of $\hat{H}^{160}$, where $\hat{H}$ denotes the Hamiltonian. Imaginary energy $0.01 \mathrm{eV}$ is added to the energy $\omega$ for smearing $\delta$-function peaks. Thin vertical line shows the highest ionization levels $9.4 \mathrm{eV}$, $7.9 \mathrm{eV}$, and $6.9 \mathrm{eV}$ (at $V=0.5,0.2$, and $0 \mathrm{eV}$ ). (b) Each orbital component $\left(3 z^{2}-1\right.$ or $x^{2}-y^{2}$ orbital) of the spectral function is drawn separately, near the highest ionization level. Thin vertical line shows the highest ionization levels, same as (a). Both the highest ionization level and the lowest affinity level consist of the $x^{2}$ $-y^{2}$ orbital and the symmetry is labeled by $\vec{k}=\left(\frac{\pi}{2}, \frac{\pi}{2}\right) \frac{1}{a}$. See also Table II.

site is most probable, due to large $U$, and the number of these pairs of sites is maximized at $x=\frac{1}{2}$. This causes an insufficient description of the ground state by a single determinant in LSNO at $x=\frac{1}{2}$.

Finally, we stress that the above stripe spin correlation induced by the anisotropy of the transfer integrals is only

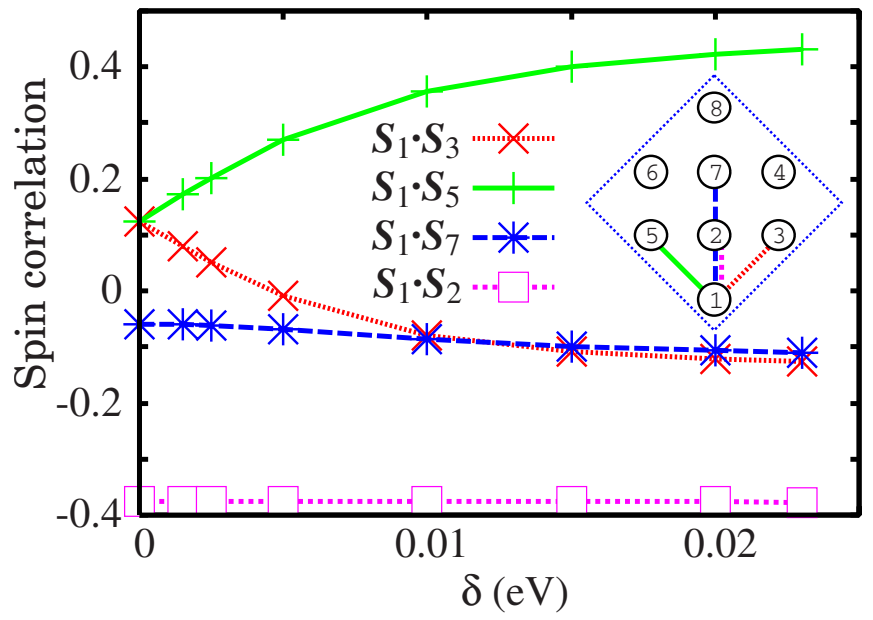

FIG. 10. (Color online) Spin correlation function $\left\langle\mathbf{S}_{i} \cdot \mathbf{S}_{j}\right\rangle$ with the anisotropy parameter $\delta$ of the second nearest hopping integrals. The value of $V$ is equal to $0.5 \mathrm{eV}$. Inset shows site location.

seen under the checkerboard-type charge order. When the anisotropy $\delta=0.02 \mathrm{eV}$ is introduced to state $S$ (see Sec. V) in the range $0 \mathrm{eV} \leqslant V \leqslant 0.4 \mathrm{eV}$, the resultant state shows the spin order of Néel order type, which is also seen in the $\delta$ $=0$ case in the range $0 \mathrm{eV} \leqslant V \leqslant 0.4 \mathrm{eV}$. The Néel order of half-filled $3 z^{2}-1$ orbitals appears under the homogeneous charge distribution, and the small $\delta$ does not affect the spin order. Under the charge ordered condition, the single electron wave function is rather localized, if not site localized. Then the transfer integrals are reduced effectively. Then the small $\delta$ changes the spin order drastically into the spin stripe.

\section{CONCLUSION}

We discussed the charge and spin order of LSNO $\left(x=\frac{1}{3}\right)$ by using the LSDA $+U$ method and those of $\operatorname{LSNO}\left(x=\frac{1}{2}\right)$ by using exact diagonalization of the double orbital extended Hubbard model derived from LDA calculations. In the exact diagonalization, charge and spin order is discussed by using charge-charge or spin-spin correlation functions. Excitation spectra of LSNO $\left(x=\frac{1}{2}\right)$ are also calculated to show that the system is insulator.

In conclusion, LSNO with $x=\frac{1}{2}$ and $\frac{1}{3}$ are both insulators with charge and spin stripe order. In both systems, diagonal hole stripes are separately located on $\mathrm{Ni}^{3+}$ site in order to reduce hole-hole interaction energy induced by intersite Coulomb interaction $V$. We discussed the important role of multiorbitals and mixing of multiple Slater determinants especially in the high-doped $x=\frac{1}{2}$ system, where the charge order is induced by the correlation energy of the intersite Coulomb interaction $V$. Charge order and the intersite Coulomb interaction $V$ are directly related to the energy gap in the excitation spectra of the system with $x=\frac{1}{2}$, spin stripe occurs only under the condition of the existence of the charge order, with the help of anisotropy $\delta$ in diagonal hopping. Thus, the spin stripe is determined by the electronic structure with smaller energy scale than that of the charge stripe. This is consistent with the observation of $T_{\mathrm{CO}}>T_{\mathrm{SO}}$. Though the spin stripe is 
related to anisotropy, the mechanism is not of Jahn-Teller type, unlike the usual ordering in transition metal oxides, because anisotropy in LSNO does not couple to the JahnTeller mode.

The stability of LNSO in different hole concentration $x$ depends sensitively upon several physical quantities, e.g., $t_{i \alpha j \beta}, \Delta, U, J, V$, and $\delta$, and we believe that we have successfully shown the new scope of the combination with the first principles electronic structure calculations and the manyelectron theory. We have developed a very useful tool for the extremely large matrix of extended Hubbard Hamiltonian, the shifted-COCG method, which will be explained in more detail elsewhere.

Last we comment on layered cuprates. The band gap of $\mathrm{La}_{2} \mathrm{CuO}_{4}(\mathrm{LCO})$ is $2 \mathrm{eV}$, narrower than $\mathrm{La}_{2} \mathrm{NiO}_{4}$, and hole doping makes the systems metallic. ${ }^{31}$ Due to these facts, the screened Coulomb interaction in LCO becomes smaller than in LSNO and the energy gain by hole hopping is more important in doped cuprates. This may be one of the reasons why the hole stripe in $\mathrm{La}_{1.48} \mathrm{Nd}_{0.4} \mathrm{Sr}_{0.12} \mathrm{CuO}_{4}$ runs in a direction along the $\mathrm{NN}$ pair and the hole concentration is one per two $\mathrm{Cu}$ sites in the stripe. ${ }^{1}$

\section{ACKNOWLEDGMENTS}

The authors benefited greatly from discussions with M. Imada, Y. Tokura, and N. Nagaosa. Calculations were done at the Supercomputer Center, Institute for Solid State Physics, University of Tokyo, and at Research Center for Computational Science, Okazaki, Japan. This work was partially supported by a Grant-in-Aid for Scientific Research in Priority Areas "Development of New Quantum Simulators and Quantum Design" (Grant No. 170640004) of The Ministry of Education, Culture, Sports, Science, and Technology, Japan.

\section{APPENDIX A: METHOD TO GET SMOOTH EIGENVECTORS WITH RESPECT TO A PARAMETER IN A HAMILTONIAN}

We use the Lanczos method and the inverse-iteration method with CG method alternatively in order to obtain the exact eigenvectors. These methods work well for obtaining ground states, but careful treatment must be taken for solving two smooth eigenvectors with respect to $V$ in Sec. V. Because both methods are the energy minimization process, iterative application of them, with no care, gives the eigenvector of lower eigenenergy than that of the aiming eigenenergy smoothly connected from the solved eigenenergy at adjacent values of $V$, though one chooses the solved eigenvector at adjacent value of $V$ as a starting vector. When the two levels are nearly degenerate, this problem becomes seriously important. In the present case, at least three energy levels cross with changing $V$ from $0 \mathrm{eV}$ to $0.5 \mathrm{eV}$, because at $V=0.5 \mathrm{eV}$, the lowest three levels are $36.7549,36.7551$, 36.7773 in units of eV (the first one is a level of state $S$ and all three levels are doubly degenerate). State $S$ locates at $36.7939 \mathrm{eV}$ at $V=0.5 \mathrm{eV}$, higher than these three levels, while state $S$ is the ground state at $V=0 \mathrm{eV}$.
The first technique to avoid this problem is to use a small number of the dimension of the submatrix tridiagonalized by the Lanczos method, since the Lanczos method has a stronger tendency to reduce a trial eigenvalue than that of inverse iteration with the CG method. We tried successfully the dimension in the range $0-80$ depending on the case. The second technique is to control the trial eigenvalue so that the residual is minimized and the trial eigenvalue does not jump, in the whole processes. Here, the residual is defined as $\|[\hat{H}$ -(trial energy)](trial vector)\|. Because increase of the residual is a sign of the transition into the lower level, once it occurs, we dispose a new (and possibly lower) trial eigenvalue.

Last, the inner product among resultant eigenvectors of respective values of the specific parameter ( $V$ in the present case) must be checked. The large value of the inner product assures the smoothness of connection to the resultant eigenvectors. In the present case, the inner product between state $S$ at $V=0 \mathrm{eV}$ and that at $V=0.5 \mathrm{eV}$ is equal to 0.54 and the inner product between state $D$ at $V=0 \mathrm{eV}$ and that at $V$ $=0.5 \mathrm{eV}$ is equal to 0.73 . These values are large enough to assure the smoothness of these eigenvectors, because no other eigenvectors at the end point $V=0.5 \mathrm{eV}$ can have larger inner product than the above respective states.

\section{APPENDIX B: WEIGHT OF THE SLATER DETERMINANTS WITH COMPLETE CHARGE ORDER, AT $V=0$}

One might have a question why state $S$ has a finite weight 0.045 of SDCCO's at $V=0$, though the state has no charge order. We conclude that the SDCCO's are not overweighted by the following discussion. Because the energy scale of the charge order is larger than that of spin order in the present system, we neglect the spin configuration in each Slater determinant, and count only the charge degrees of freedom. Strong $U$ inhibits the configurations with site occupation more than 2 and 0 and there exist ${ }_{8} \mathrm{C}_{4}=70$ types of the charge configurations, where the site occupations are restricted to be 1 or 2. A number of charge configurations with complete checkerboard-type order are equal to 2. Assuming that all configurations have the same weight due to the absence of charge order, resultant weight of SDCCO's is equal to $\frac{2}{70}-0.029$, which is consistent with the above 0.045 .

\section{APPENDIX C: GAP WIDTH IN THE BULK LIMIT}

First we discuss the value of the energy gap $0.9 \mathrm{eV}$ in two ways, applying two extreme approximations, and show how intersite Coulomb interaction opens the energy gap. One of two approximations is such that HIL and LAL are approximated by a single particle excitation related to an extended hole and/or electron, and the other is such that they are approximated by a single particle excitation of a site localized hole and/or electron. In the actual picture, excitations are described by dressed quasiparticle and/or hole and not by a single particle and/or hole, as is discussed later in the present section. 
We begin with the case that HIL/LAL is approximated by a single particle excitation of an extended state. Due to large splitting $\Delta=0.97 \mathrm{eV}$ between the $3 z^{2}-1$ and the $x^{2}-y^{2}$ orbital hole is doped into the $x^{2}-y^{2}$ orbital. Therefore, low energy excitation mostly consists of the $x^{2}-y^{2}$ orbital and we neglect the $3 z^{2}-1$ orbital in the present paragraph. The single electron wave functions of HIL and LAL belong to the same symmetry group labeled $\boldsymbol{k}=\left( \pm \frac{\pi}{2}, \pm \frac{\pi}{2}\right) \frac{1}{a}$ as in Table II. By unitary transformation, four plain waves labeled $\boldsymbol{k}$ $=\left( \pm \frac{\pi}{2}, \pm \frac{\pi}{2}\right) \frac{1}{a}$ change the forms into four checkerboard-type wave functions $\cos \left(\frac{\pi}{2} x\right) \cos \left(\frac{\pi}{2} y\right), \quad \sin \left(\frac{\pi}{2} x\right) \sin \left(\frac{\pi}{2} y\right)$, $\cos \left(\frac{\pi}{2} x\right) \sin \left(\frac{\pi}{2} y\right), \sin \left(\frac{\pi}{2} x\right) \cos \left(\frac{\pi}{2} y\right)$. These four wave functions give the two types of charge distribution. One type is shown in Fig. 6(b) and the other is obtained with exchanging hole and nonhole sites. Two wave functions $\cos \left(\frac{\pi}{2} x\right) \cos \left(\frac{\pi}{2} y\right)$ and $\sin \left(\frac{\pi}{2} x\right) \sin \left(\frac{\pi}{2} y\right)$ do not interact with on-site Coulomb interaction $U$, because $\left[\cos ^{2}\left(\frac{\pi}{2} x\right) \cos ^{2}\left(\frac{\pi}{2} y\right)\right]\left[\sin ^{2}\left(\frac{\pi}{2} x\right) \sin ^{2}\left(\frac{\pi}{2} y\right)\right]$ $=16 \sin ^{2}(\pi x) \sin ^{2}(\pi y)=0$ on each lattice point. Instead, the interaction between them with intersite Coulomb interaction $V$ is roughly estimated as $\frac{1}{n} \times \frac{1}{n} \times 4 V \times n=\frac{4 V}{n}$, where $n$ $=($ the number of sites whose amplitude is finite $)=4$ in the present case. This is occupied and unoccupied splitting. Assuming $\cos \left(\frac{\pi}{2} x\right) \cos \left(\frac{\pi}{2} y\right)$ is occupied, then $\sin \left(\frac{\pi}{2} x\right) \sin \left(\frac{\pi}{2} y\right)$ is unoccupied and vice versa. The same situation occurs for another pair of wave functions $\cos \left(\frac{\pi}{2} x\right) \sin \left(\frac{\pi}{2} y\right)$ and $\sin \left(\frac{\pi}{2} x\right) \cos \left(\frac{\pi}{2} y\right)$. Thus, the system becomes insulator with gap roughly estimated as $\frac{4 V}{n}=0.5 \mathrm{eV}$, except $V=0 \mathrm{eV}$, where all the occupied and unoccupied states are degenerate [see Fig. 9(b)]. Though the correlation induced by intersite Coulomb interaction causes the splitting between occupied and unoccupied states (the energy gap), this mechanism is different from the normal Mott insulator, induced by on-site Coulomb interaction $U .{ }^{29} \mathrm{In}$ addition to that, there is another difference between the current mechanism and that of the Mott insulator; the wave function of HIL and LAL have checkerboardtype charge order, not sharing the center of charge distribution in the current mechanism, unlike those that share the center of charge distribution in the Mott insulator. However, there is a problem with this estimation of the gap. Following the present estimation, the energy gap vanishes in the bulk limit, because the gap is inversely proportional to the system size.

In the next estimation, HIL and/or LAL excitation is approximated by a single particle excitation of site localized hole and/or electron. Neglecting hopping integrals, the ground state of the present Hamiltonian consists of four $\mathrm{Ni}^{2+}$ (spin triplet, $u^{1} v^{1}$ ) and four doublet $\mathrm{Ni}^{3+}$ (spin doublet, $u^{1}$ ) located on a respective sublattice, where $u$ and $v$ denote $3 z^{2}-1$ and $x^{2}-y^{2}$ orbital, respectively. All these components are assigned to the Slater determinants with complete checkerboard-type charge order (SDCCO's) in Sec. V. Then the LAL is the $x^{2}-y^{2}$ orbital of $\mathrm{Ni}^{3+}$ and the HIL is the $x^{2}$ $-y^{2}$ orbital of $\mathrm{Ni}^{2+}$ (both have a majority spin). Consequently, the energy gap is estimated as $\left\{\left[E\left(\mathrm{Ni}^{2+}\right)\right]+8 \mathrm{~V}\right.$ $\left.-\left[E\left(\mathrm{Ni}^{3+}\right)+4 V\right]\right\}-\left\{E\left(\mathrm{Ni}^{2+}\right)-E\left(\mathrm{Ni}^{3+}\right)\right\}=4 V=0.5 \mathrm{eV}$, where the $E(\cdot)$ denotes the ground-state energy of the $\mathrm{Ni}$ ion for each ionization state. This is an exact solution even in the bulk limit.

The result of the present calculation is in the intermediate region of the above two estimations; the calculated energy gap $0.9 \mathrm{eV}$ is greater than $0.5 \mathrm{eV}$ in extreme cases by the extended HIL and/or LAL approximation and less than $2.0 \mathrm{eV}$ by the site localized HIL and/or LAL approximation. Therefore, the calculated excitations (with respect to state $D$ at $V=0.5 \mathrm{eV}$ in Sec. V) are well described by the wave function in the intermediate region between site localized and extended states. The split between occupied and unoccupied states occurs due to long-ranged (intersite) Coulomb interaction, though they are not sharing the center and do not have large overlap.

Finally, we discuss the bulk limit of the energy gap in the present calculation. Taking the limit such that transfer integrals go to infinity, the system becomes paramagnetic metal. Taking the limit such that transfer integrals go to zero the system becomes an antiferromagnetic insulator. Therefore, there exists a critical transfer integral where the bulk limit of the system changes from insulator (metal) to metal (insulator). The high weight of the Slater determinant with complete checkerboard-type charge order $(>0.4$ at $V=0.5 \mathrm{eV}$, as in Fig. 7) strongly suggests that the present choice of transfer integrals makes the bulk limit insulator, and that is consistent with the real $\operatorname{LSNO}\left(x=\frac{1}{2}\right)$.
${ }^{1}$ J. M. Tranquada, B. J. Sternlieb, J. D. Axe, Y. Nakamura, and S. Uchida, Nature (London) 375, 561 (1995).

${ }^{2}$ J. M. Tranquada, D. J. Buttrey, V. Sachan, and J. E. Lorenzo, Phys. Rev. Lett. 73, 1003 (1994).

${ }^{3}$ T. Kimura, K. Hatsuda, Y. Ueno, R. Kajimoto, H. Mochizuki, H. Yoshizawa, T. Nagai, Y. Matsui, A. Yamazaki, and Y. Tokura, Phys. Rev. B 65, 020407(R) (2001).

${ }^{4}$ K. Miyagawa, A. Kawamoto, and K. Kanoda, Phys. Rev. B 62, R7679 (2000).

${ }^{5}$ Y. Takano, K. Hiraki, H. M. Yamamoto, T. Nakamura, and T. Takahashi, J. Phys. Chem. Solids 62, 393 (2001).

${ }^{6}$ H. Seo, C. Hotta, and H. Fukuyama, Chem. Rev. (Washington, D.C.) 104,5005 (2004).

${ }^{7}$ V. Sachan, D. J. Buttrey, J. M. Tranquada, J. E. Lorenzo, and G.
Shirane, Phys. Rev. B 51, 12742 (1995).

${ }^{8}$ J. M. Tranquada, D. J. Buttrey, and V. Sachan, Phys. Rev. B 54, 12318 (1996).

${ }^{9}$ H. Yoshizawa, T. Kakeshita, R. Kajimoto, T. Tanabe, T. Katsufuji, and Y. Tokura, Phys. Rev. B 61, R854 (2000).

${ }^{10}$ R. Kajimoto, K. Ishizaka, H. Yoshizawa, and Y. Tokura, Phys. Rev. B 67, 014511 (2003).

${ }^{11}$ R. J. Cava, B. Batlogg, T. T. Palstra, J. J. Krajewski, W. F. Peck, Jr., A. P. Ramirez, and L. W. Rupp, Jr., Phys. Rev. B 43, 1229 (1991).

${ }^{12}$ J. Zaanen and O. Gunnarsson, Phys. Rev. B 40, 7391 (1989).

${ }^{13}$ T. Hotta and E. Dagotto, Phys. Rev. Lett. 92, 227201 (2004).

${ }^{14}$ V. J. Emery and S. A. Kivelson, Physica C 209, 597 (1993).

${ }^{15}$ Y. Ohta, K. Tsutsui, W. Koshibae, and S. Maekawa, Phys. Rev. B 
50, 13594 (1994).

${ }^{16}$ M. Raczkowski, R. Frésard, and A. M. Oleś, Phys. Rev. B 73, 094429 (2006).

${ }^{17}$ V. I. Anisimov, J. Zaanen, and O. K. Andersen, Phys. Rev. B 44, 943 (1991).

${ }^{18}$ A. I. Liechtenstein, V. I. Anisimov, and J. Zaanen, Phys. Rev. B 52, R5467 (1995).

${ }^{19}$ O. K. Andersen, Phys. Rev. B 12, 3060 (1975).

${ }^{20}$ O. K. Andersen and O. Jepsen, Phys. Rev. Lett. 53, 2571 (1984).

${ }^{21}$ Other formulation for the matrix elements of Coulomb interaction is given in A. M. Oles, Phys. Rev. B 28, 327 (1983).

22 A. E. Bocquet, T. Mizokawa, T. Saitoh, H. Namatame, and A. Fujimori, Phys. Rev. B 46, 3771 (1992).

${ }^{23}$ M. Imada, A. Fujimori, and Y. Tokura, Rev. Mod. Phys. 70, 1039 (1998).

${ }^{24}$ J. Rodríguez-Carvajal, M. T. Fernández-Díaz, and J. L. Martínez, J. Phys.: Condens. Matter 3, 3215 (1991).

${ }^{25}$ T. Ido, K. Magoshi, H. Eisaki, and S. Uchida, Phys. Rev. B 44, 12094 (1991).
${ }^{26}$ The calculated ground state by the LDA result for $x=0$ is the paramagnetic metal without orbital polarization.

${ }^{27}$ Y. Nohara, A. Yamasaki, S. Kobayashi, and T. Fujiwara, Phys. Rev. B 74, 064417 (2006).

${ }^{28}$ R. Takayama, T. Hoshi, T. Sogabe, S.-L. Zhang, and T. Fujiwara, Phys. Rev. B 73, 165108 (2006).

${ }^{29}$ The ground state in the range $V<0.41 \mathrm{eV}$ (state $S$ ) is insulator with no charge order, such that the charge gap opens due to on-site Coulomb interaction $U$.

${ }^{30}$ More physically meaningful comparison is made for the normalized values of $\left\langle\mathbf{S}_{1} \cdot \mathbf{S}_{2}\right\rangle / \sqrt{\left\langle\mathbf{S}_{1}{ }^{2}\right\rangle\left\langle\mathbf{S}_{2}{ }^{2}\right\rangle},-0.08\left(x^{2}-y^{2}\right.$ orbital system), and -0.29 (LSNO).

${ }^{31}$ Where doping level $x<0.05, \mathrm{La}_{2-x} \mathrm{Sr}_{x} \mathrm{CuO}_{4}$ remains insulator and its charge order shows diagonal stripe. S. Wakimoto, G. Shirane, Y. Endoh, K. Hirota, S. Ueki, K. Yamada, R. J. Birgeneau, M. A. Kastner, Y. S. Lee, P. M. Gehring, and S. H. Lee, Phys. Rev. B 60, R769 (1999). 